\title{
Signatures of bilayer Wigner crystals in a transition metal dichalcogenide heterostructure
}

You Zhou ${ }^{1,2}$, Jiho Sung ${ }^{1,2}$, Elise Brutschea ${ }^{1}$, Ilya Esterlis ${ }^{2}$, Yao Wang ${ }^{2,3}$, Giovanni Scuri², Ryan J. Gelly $^{2}$, Hoseok Heo ${ }^{1,2}$, Takashi Taniguchi ${ }^{4}$, Kenji Watanabe ${ }^{4}$, Gergely Zaránd ${ }^{5}$, Mikhail D. Lukin $^{2}$, Philip Kim ${ }^{2,6}$, Eugene Demler ${ }^{2 \dagger}$ \& Hongkun Park ${ }^{1,2 \dagger}$

${ }^{1}$ Department of Chemistry and Chemical Biology, Harvard University, Cambridge, MA 02138, USA

${ }^{2}$ Department of Physics, Harvard University, Cambridge, MA 02138, USA

${ }^{3}$ Department of Physics and Astronomy, Clemson University, Clemson, SC 29634, USA

${ }^{4}$ National Institute for Materials Science, 1-1 Namiki, Tsukuba 305-0044, Japan

${ }^{5}$ MTA-BME Quantum Dynamics and Correlations Research Group, Institute of Physics, Budapest University of Technology and Economics Budafoki ut 8., H-1111 Budapest, Hungary

${ }^{6}$ John A. Paulson School of Engineering and Applied Sciences, Harvard University, Cambridge, MA 02138, USA

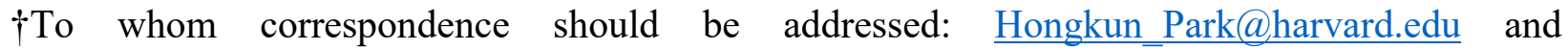
demler@physics.harvard.edu 
A Wigner crystal, a regular electron lattice arising from strong correlation effects ${ }^{1-6}$, is one of the earliest predicted collective electronic states. This many-body state exhibits quantum and classical phase transitions ${ }^{7}$ and has been proposed as a basis for quantum information processing applications ${ }^{8,9}$. In semiconductor platforms, two-dimensional Wigner crystals have been observed under magnetic field ${ }^{10-17}$ or moiré-based lattice potential ${ }^{18-21}$ where the electron kinetic energy is strongly suppressed. Here, we report bilayer Wigner crystal formation without a magnetic or confinement field in atomically thin MoSe $\mathrm{M}_{2}$ bilayers separated by hexagonal boron nitride. We observe optical signatures of robust correlated insulating states formed at symmetric (1:1) and asymmetric (4:1 and 7:1) electron doping of the two $\mathrm{MoSe}_{2}$ layers at cryogenic temperatures. We attribute these features to the bilayer Wigner crystals formed from two commensurate triangular electron lattices in each layer, stabilized via inter-layer interaction ${ }^{22,}{ }^{23}$. These bilayer Wigner crystal phases are remarkably stable and undergo quantum and thermal melting transitions above a critical electron density of up to $6 \times 10^{12} \mathrm{~cm}^{-2}$ and at temperatures of $\sim 40 \mathrm{~K}$. Our results demonstrate that atomically thin semiconductors provide a promising new platform for realizing strongly correlated electronic states, probing their electronic and magnetic phase transitions, and developing novel applications in quantum electronics and optoelectronics ${ }^{24-28}$.

Atomically thin heterostructures made of graphene and transition metal dichalcogenide (TMD) monolayers can host a variety of correlated electronic states ${ }^{29-33}$. Recent advances in materials growth and heterostructure fabrication have enabled the preparation of high-quality heterostructures with minimal disorder ${ }^{34-38}$. The large effective masses of charge carriers ${ }^{39,40}$ and the weak Coulomb screening in TMDs suppress the Fermi energy and enhance electron 
interactions, thus facilitating the realization of correlated electronic phases at much higher electron density $^{18-21,41}$ in comparison to other semiconductors such as GaAs ${ }^{10-14,42,43}$. Furthermore, owing to their strong excitonic response that is sensitive to the spin and charge states of the material, the electrical properties of TMD heterostructures can be optically detected ${ }^{36,37,44-46}$. Indeed, experiments on TMD heterostructures with moiré superlattices have found optical signatures of correlated electron solids ${ }^{18-21}$, sometimes called generalized Wigner crystals $^{4,47}$, aided by the underlying moiré potential at particular electron fillings.

In this work, we create a high-quality $\mathrm{MoSe}_{2}$ bilayer heterostructure and optically investigate the formation of bilayer Wigner crystals at zero magnetic field and high electron densities. The heterostructure consists of two $\mathrm{MoSe}_{2}$ monolayers (nominal $0^{\circ}$ twist angle) separated by a 1-nm thick hexagonal boron nitride (hBN) layer (Fig. 1a \& b, see methods ${ }^{48}$ ). The carrier density in $\mathrm{MoSe}_{2}$ layers can be independently controlled by the external gates. Figure 1c shows representative photoluminescence (PL) spectra collected from the $\mathrm{MoSe}_{2} / \mathrm{hBN} / \mathrm{MoSe}_{2}$ region under various gate configurations at $4 \mathrm{~K}$. Without external gating, the PL spectrum is dominated by sharp emission from the neutral intra-layer exciton $\left(\mathrm{X}_{0}\right)$ and a much weaker signal from the charged exciton $\left(\mathrm{X}_{\mathrm{T}}\right)$. This behavior is similar to high-quality intrinsic monolayers ${ }^{49}$, indicating that both $\mathrm{MoSe}_{2}$ layers maintain their direct bandgap (Extended Data. Fig. 1). By doping one layer selectively while keeping the other intrinsic, we find that the neutral excitons in the top and bottom layer are nearly degenerate in energy (within $0.5 \mathrm{meV}$ : Fig. $1 \mathrm{c}$ ). This observation suggests minimal and near-symmetric strain buildup in the two layers that is different from previous studies ${ }^{32}$, facilitating the formation of bilayer Wigner crystals. 
Due to the near-degeneracy of the exciton energies in the bilayer, we can characterize the gatedependent charge states of the system by measuring the reflectance of $\mathrm{X}_{0}$, which is sensitive to the free carrier concentration in $\mathrm{MoSe}_{2}{ }^{36,37,44-46}$ (Fig. 1d, see also Extended Data Fig. 1). Along the line of $V_{\mathrm{tg}}=-\alpha V_{\mathrm{bg}}\left(\alpha\right.$ is the ratio between the top and bottom hBN thickness, $\left.\alpha=d_{\mathrm{tg}} / d_{\mathrm{bg}}\right)$, we observe strong $\mathrm{X}_{0}$ reflectance, indicating that both layers are intrinsic $((i, i)$ in Fig. $1 \mathrm{~d})$. When $V_{\mathrm{tg}}$ and $V_{\mathrm{bg}}$ are of the same polarity, both $\mathrm{MoSe}_{2}$ layers are doped and the reflectance is minimized. Importantly, the charge map in Fig. 1d demonstrates full control over the carrier densities in each $\mathrm{MoSe}_{2}$ layer.

Such precise control enables the detailed interrogation of the electronic phases in the heterostructure as a function of charge density. We focus on the $(n, n)$ region, where both $\mathrm{MoSe}_{2}$ layers are electron-doped, and map out the $\mathrm{X}_{0}$ reflectance in finer voltage steps (Fig. 2a, corresponding to the dashed box in Fig. 1d). Remarkably, we observe several strong $\mathrm{X}_{0}$ reflectance features, denoted as I, II, III, and IV in Fig. 2a, which exist at particular $V_{\mathrm{tg}} / V_{\mathrm{bg}}$ ratios and diminish at higher voltages. These features are also observable in PL measurements for neutral excitons $\mathrm{X}_{0}$ (Fig. 2b) and charged excitons $\mathrm{X}_{\mathrm{T}}$ (Extended Data Fig. 1). Because the reflectance and PL intensity of $\mathrm{X}_{0}$ depends sensitively on the presence of free carriers in the system ${ }^{36,37,44-46}$, these observations indicate that at these specific $V_{\mathrm{tg}} / V_{\mathrm{bg}}$ ratios, unexpected insulating states are formed in the bilayer even though both layers are doped with electrons.

Next, we examine the existence conditions for these insulating states. From the PL data and a capacitance model (see Methods), we find that the features I, II, III, and IV correspond to electron filling ratios between the top and bottom layers of $n_{\mathrm{t}}: n_{\mathrm{b}}=1.0( \pm 0.2): 1,4.3( \pm 0.4): 1,7.4( \pm 0.7): 1$, and 
1:4.0( \pm 0.4$)$, respectively $\left(n_{\mathrm{t}}\right.$ and $n_{\mathrm{b}}$ represents the electron density in the top and bottom layer, respectively). In Fig. 2c we plot the integrated $\mathrm{X}_{0}$ PL intensity as a function of $n_{\mathrm{t}}$ for different $n_{\mathrm{b}}$ values. We observe multiple prominent peaks, corresponding to the features in Fig. 2b, whose positions shift with varying $n_{\mathrm{b}}$. This is in stark contrast with the situation for large $n_{\mathrm{b}}(e . g$., $4.2 \times$ $10^{12} \mathrm{~cm}^{-2}$ in Fig. 2c) or what is observed in monolayers: in those situations, the PL intensity of $\mathrm{X}_{0}$ decreases monotonically when the doping is increased. We note that the linewidths of the neutral exciton along features I, II, III, and IV are much narrower than those at other $n_{\mathrm{t}}: n_{\mathrm{b}}$ ratios or from monolayers of the similar electron density (Fig.2d and Extended Data Fig. 1). These observations indicate that along features I, II, III, and IV there is less dephasing stemming from exciton-free carrier interactions ${ }^{46}$, thus confirming that these states are more insulating ${ }^{36,37,44-46}$.

To disentangle the density-dependent behaviour of the insulating states from the intrinsic gate dependence of $\mathrm{MoSe}_{2}$ monolayers ${ }^{36,37,44-46}$ (Extended Data Fig. 2), we introduce a dimensionless parameter $\delta\left(n_{\mathrm{t}}, n_{\mathrm{b}}\right)$ that characterizes the inter-layer coupling:

$$
\delta\left(n_{\mathrm{t}}, n_{\mathrm{b}}\right) \equiv\left(I_{0}\left(n_{\mathrm{t}}, n_{\mathrm{b}}\right)-I_{\mathrm{t}}\left(n_{\mathrm{t}}\right)-I_{\mathrm{b}}\left(n_{\mathrm{b}}\right)\right) / I_{0}(0,0) .
$$

Here $I_{0}\left(n_{\mathrm{t}}, n_{\mathrm{b}}\right)$ is the device's total PL intensity from $\mathrm{X}_{0}$, and $I_{\mathrm{t}}\left(n_{\mathrm{t}}\right)\left(I_{\mathrm{b}}\left(n_{\mathrm{b}}\right)\right)$ is the PL intensity of $\mathrm{X}_{0}$ from only the top (bottom) layer when its electron density is $n_{\mathrm{t}}\left(n_{\mathrm{b}}\right)$. In a situation where there is no interaction between the two $\mathrm{MoSe}_{2}$ layers, the total emission should simply be the sum of individual PL from both layers, and $\delta\left(n_{\mathrm{t}}, n_{\mathrm{b}}\right)$ should be zero. The value of $\delta\left(n_{\mathrm{t}}, n_{\mathrm{b}}\right)$ therefore quantifies the interactions between the two layers.

Figure 3a presents a two-dimensional (2D) map of $\delta\left(n_{\mathrm{t}}, n_{\mathrm{b}}\right)$ extracted from our experimental data in Fig. 2 (see SI for details): it clearly shows the enhancement of $\delta$ along the particular filling ratios 
$n_{\mathrm{t}}: n_{\mathrm{b}}$ compared with other values. Figure $3 \mathrm{~b}$ shows $\delta$ as a function of total carrier density $n=n_{\mathrm{t}}+$ $n_{\mathrm{b}}$, measured along features I-IV in Fig. 2, i.e. $n_{\mathrm{t}}: n_{\mathrm{b}}=1: 1,4: 1,7: 1$, and 1:4. The values of $\delta$ reach their maximum at around $10^{12} \mathrm{~cm}^{-2}$ and diminish to zero at higher densities.

Next, we characterize the temperature dependence of these insulating states. Figures $3 \mathrm{c}$ and $3 \mathrm{~d}$ show the $2 \mathrm{D}$ maps of $\delta\left(n_{\mathrm{t}}, n_{\mathrm{b}}\right)$ at $23 \mathrm{~K}$ and $35 \mathrm{~K}$, which display weaker insulating features compared to $4 \mathrm{~K}$ (additional temperature points are shown in Extended Data Figs. $3 \& 4$ ). From these temperature-dependent studies, we find that the 1:1, 4:1 and 7:1 features exhibit different thermodynamic stabilities and persist up to $40 \mathrm{~K}, 30 \mathrm{~K}$, and $23 \mathrm{~K}$, respectively. It is important to note that the critical density for the insulating states also decreases with increasing temperatures, as will be discussed below.

We attribute the unexpected insulating states in Figs. 2 and 3 to the formation of bilayer Wigner crystals. As discussed previously, the large effective masses of charge carriers ${ }^{39,40}$ and weak Coulomb screening in TMD heterostructure are conducive for the Wigner crystal formation ${ }^{41}$. Furthermore, the Fermi energy of a bilayer system is reduced compared to a single layer system with a similar total density because the electron density is divided into two layers (Fig. 4a). Finally, previous theoretical studies have predicted that the inter-layer Coulomb interactions stabilize a bilayer Wigner crystal phase by lowering the system's overall potential energy as compared to two uncoupled single-layer Wigner crystals ${ }^{22}$.

To understand the formation of a bilayer Wigner crystal at particular filling ratios, we consider a simple physical model where intra-layer electron-electron interactions are much stronger than 
those between the layers. In this weak coupling regime, the inter-layer interaction can be treated as a perturbation to two triangular Wigner crystals, and the stable commensurate bilayer lattices form only at particular $n_{\mathrm{t}}: n_{\mathrm{b}}$ values. Geometrical considerations dictate that the first few $n_{\mathrm{t}}: n_{\mathrm{b}}$ to form such commensurate lattices are 1:1, 3:1, 4:1, and 7:1 (Fig. $4 \mathrm{~b}$ and SI). We note that in the limit of vanishing inter-layer distance, the $n_{\mathrm{t}}: n_{\mathrm{b}}$ values should not be important for the stability of Wigner crystals ${ }^{50,51}$.

To describe our experimental data in a more quantitative fashion, we investigate the system's stability as a function of inter-layer coupling, considering only the potential energy ${ }^{52}$, and numerically optimize the lattice geometry for various $n_{\mathrm{t}}: n_{\mathrm{b}}$ values. From such analysis, we find that for $n_{\mathrm{t}}: n_{\mathrm{b}}$ values of $1: 1,3: 1,4: 1,7: 1$, the bilayer Wigner crystals have lower energies compared to the decoupled ones due to the commensurability of the two triangular lattices (the layer symmetry dictates that the filling ratios of e.g., $4: 1$ and $1: 4$ are equivalent). In contrast, for $n_{\mathrm{t}}: n_{\mathrm{b}}$ values that lead to incommensurate stacking of two monolayer Wigner crystals (e.g., 2:1 and 5:1), the bilayer lattices have higher energies than the decoupled layers in the weak coupling regime (Extended Data Figs. 5 \& 6). The absence of 3:1, 1:3, and 1:7 features in our experiments suggests that the Wigner crystals at those particular ratios may be unstable in the $\mathrm{MoSe}_{2} / \mathrm{hBN} / \mathrm{MoSe}_{2} \mathrm{system}$ for reasons that we have not accounted for, such as non-ideal contact to TMDs at small gate voltages. Additional experimental and theoretical studies are needed to clarify these issues.

We attribute the density and temperature dependence of the insulating states (Figs. 2 and 3) to the quantum and thermal melting of a Wigner crystal. With increasing electron density, the kinetic energy of the electrons increases faster than the potential energy, eventually melting the Wigner 
crystal. Notably, at $4 \mathrm{~K}$, the Fermi energy of the electrons near the critical density ( $E_{\mathrm{F}} \sim 10 \mathrm{meV}$ near $n_{t}\left(\right.$ or $\left.n_{b}\right) \sim 3 \times 10^{12} \mathrm{~cm}^{-2}$, based on the electron effective mass $\left.{ }^{53-55}\right)$ is much larger than the thermal energy $\left(k_{\mathrm{B}} T \sim 0.3 \mathrm{meV}\right)$, and thus the system should exhibit a quantum melting behavior.

Figure $4 \mathrm{c}$ shows a 2D map of $\delta\left(n_{\mathrm{t}}, n_{\mathrm{b}}\right)$ for $n_{\mathrm{t}}: n_{\mathrm{b}}=1: 1$ as a function of total electron density $n=n_{\mathrm{t}}$ $+n_{\mathrm{b}}$ and temperature $T$ (see SI for the discussions of $n_{\mathrm{t}}: n_{\mathrm{b}}=4: 1$ and 7:1 cases). Taking $\delta>0$ as a proxy for the interaction-driven insulating state, the phase boundary between an electron solid and a liquid can be approximated by the $\delta=0$ line (dashed line in Fig. 4c). Such an experimentally extracted phase boundary indeed resembles the generic theoretical phase diagram of a Wigner crystal $^{56}$, which exhibits a dome shape due to the quantum and thermal phase transitions.

To obtain a qualitative understanding of the bilayer Wigner crystal phase diagram, we calculate the phonon spectrum for the classical bilayer Wigner crystal and estimate the melting curve via a modified Lindemann criterion appropriate to two dimensions ${ }^{28,57}$. Specifically, we assume that the melting occurs when the ratio of root-mean-square fluctuations of the nearest-neighbor electron distance to the lattice constant exceeds a critical value $\gamma$ (see SI for details). Here we focus on the ratio 1:1. Previous studies have found the Lindemann parameter $(\gamma)$ for the bilayer crystal is in fact dependent on the inter-layer separation ${ }^{58}$ and we therefore treat it is a fitting parameter. Matching to the $T=4 \mathrm{~K}$ critical density, we find $\gamma \sim 0.56$. We contrast this with the monolayer Wigner crystal, where numerical simulations have shown $\gamma \sim 0.1-0.3^{28,59}$. The large value of $\gamma$ in our system, which is a direct result of the high critical density observed in the experiment, indicates the enhanced stability of the bilayer crystal. 
As shown in Fig. 4d, a melting curve calculated with a modified Lindemann criterion tracks well with the experimentally determined $\delta\left(n_{\mathrm{t}}, n_{\mathrm{b}}\right)$ map in Fig. 4c. The dashed curve in Fig. $4 \mathrm{~d}$ terminates at a critical density $\sim 1.4 \times 10^{12} \mathrm{~cm}^{-2}$, at which point theory predicts the staggered triangular lattice structure becomes unstable, giving way to a sequence of structural transitions ${ }^{7}{ }^{57}$ (see SI and Extended Data Fig. 5). We emphasize that while the fitting procedure described here yields a melting curve compatible with experimental data, further experimental and theoretical work is needed to understand the mechanism for the dramatic enhancement of stability of the bilayer crystal. We also remark that the temperature scale for melting determined by the experimental data in Fig. 4c is most likely an overestimate of the true melting temperature. Instead, the $\delta=0$ line in Fig. $4 \mathrm{c}$ is more precisely understood as a crossover to the insulating regime, which would precede the establishment of long-range order. The robustness of insulating states for specific $n_{t}: n_{b}$ values suggests that even in the presence of disorder ${ }^{60}$, the Wigner crystal domains are still sufficiently large to establish local crystalline order. Combined optical and transport measurements ${ }^{61-64}$ should provide additional information on the interplay between the crystalline correlations and disorder.

The observation of bilayer Wigner crystals in atomically thin semiconductor heterostructures without a magnetic or moiré-confinement field opens up exciting avenues for creating, studying, and manipulating the collective quantum phases of electrons ${ }^{61-66}$. With electrically tunable carrier density and coupling strength, these devices should enable new studies of quantum and classical phase transitions in engineered many-body correlated systems ${ }^{65,66}$. Unlike a single layer Wigner crystal, which has only one stable lattice structure, bilayer Wigner crystals exhibit a rich structural phase diagram depending on their filling ratios and coupling strength (Extended Data Figs. 5 \& 6) and possess unique collective modes such as optical phonons ${ }^{52,57,67,68}$. In addition to providing an 
experimental platform to investigate the phase transitions of exotic electronic phases in the quantum regime, our devices hold promise for applications in quantum computing ${ }^{8}$ and simulation $^{69}$ as well. The Wigner crystals realized in atomically thin semiconductors can be controlled and probed both optically and electrically, and can be readily integrated with functional substrates to create on-chip devices for quantum simulations ${ }^{69-71}$. One particularly intriguing direction involves the realization and exploration of tunable spin Hamiltonians that could be used for creating exotic spin states ${ }^{69-72}$. In particular, the strong spin-orbit coupling and valleydependent selection rules in TMD heterostructures enable optical detection and manipulation of the electronic spin states ${ }^{73}$, opening up exciting new directions in simulating and manipulating spin dynamics in various lattice geometries ${ }^{74,75}$.

\section{References:}

1. Wigner, E. On the interaction of electrons in metals. Phys. Rev. 46, 1002-1011 (1934).

2. Grimes, C.C. \& Adams, G. Evidence for a liquid-to-crystal phase transition in a classical, two-dimensional sheet of electrons. Phys. Rev. Lett. 42, 795-798 (1979).

3. Porras, D. \& Cirac, J.I. Quantum manipulation of trapped ions in two dimensional Coulomb crystals. Phys. Rev. Lett. 96, 250501 (2006).

4. Wu, C., Bergman, D., Balents, L. \& Das Sarma, S. Flat bands and Wigner crystallization in the honeycomb optical lattice. Phys. Rev. Lett. 99, 070401 (2007).

5. Deshpande, V.V. \& Bockrath, M. The one-dimensional Wigner crystal in carbon nanotubes. Nat. Phys. 4, 314-318 (2008).

6. Shapir, I. et al. Imaging the electronic Wigner crystal in one dimension. Science 364, 870 (2019).

7. Monarkha, Y. \& Kono, K. Two-dimensional Coulomb liquids and solids, Vol. 142. (Springer Science \& Business Media, 2013).

8. Platzman, P.M. \& Dykman, M.I. Quantum computing with electrons floating on liquid helium. Science 284, 1967 (1999). 
9. Antonio, B., Bayat, A., Kumar, S., Pepper, M. \& Bose, S. Self-assembled Wigner crystals as mediators of spin currents and quantum information. Phys. Rev. Lett. 115, 216804 (2015).

10. Andrei, E.Y. et al. Observation of a magnetically induced Wigner solid. Phys. Rev. Lett. 60, 2765-2768 (1988).

11. Willett, R.L. et al. Termination of the series of fractional quantum hall states at small filling factors. Phys. Rev. B 38, 7881-7884 (1988).

12. Jiang, H.W. et al. Quantum liquid versus electron solid around v=1/5 Landau-level filling. Phys. Rev. Lett. 65, 633-636 (1990).

13. Manoharan, H.C., Suen, Y.W., Santos, M.B. \& Shayegan, M. Evidence for a Bilayer Quantum Wigner Solid. Phys. Rev. Lett. 77, 1813-1816 (1996).

14. Wang, Z. et al. Unequal layer densities in bilayer Wigner crystal at high magnetic fields. Phys. Rev. B 85, 195408 (2012).

15. Ma, M.K. et al. Thermal and quantum melting phase diagrams for a magnetic-field-induced Wigner solid. Phys. Rev. Lett. 125, 036601 (2020).

16. Hatke, A.T. et al. Microwave spectroscopy of the low-filling-factor bilayer electron solid in a wide quantum well. Nat. Commun. 6, 7071 (2015).

17. Tutuc, E., Melinte, S., De Poortere, E.P., Pillarisetty, R. \& Shayegan, M. Role of density imbalance in an interacting bilayer hole system. Phys. Rev. Lett. 91, 076802 (2003).

18. Regan, E.C. et al. Mott and generalized Wigner crystal states in $\mathrm{WSe}_{2} / \mathrm{WS}_{2}$ moiré superlattices. Nature 579, 359-363 (2020).

19. $\mathrm{Xu}, \mathrm{Y}$. et al. Abundance of correlated insulating states at fractional fillings of $\mathrm{WSe}_{2} / \mathrm{WS}_{2}$ moiré superlattices. arXiv:2007.11128 (2020).

20. Huang, X. et al. Correlated insulating states at fractional fillings of the $\mathrm{WS}_{2} / \mathrm{WSe}_{2}$ moiré Lattice. arXiv:2007.11155 (2020).

21. Jin, C. et al. Stripe phases in $\mathrm{WSe}_{2} / \mathrm{WS}_{2}$ moire superlattices. arXiv:2007.12068 (2020).

22. Świerkowski, L., Neilson, D. \& Szymański, J. Enhancement of Wigner crystallization in multiple-quantum-well structures. Phys. Rev. Lett. 67, 240 (1991).

23. Rapisarda, F. \& Senatore, G. Diffusion Monte Carlo study of electrons in two-dimensional layers. Aust. J. Phys. 49, 161-182 (1996). 
24. Kosterlitz, J.M. \& Thouless, D.J. Ordering, metastability and phase transitions in twodimensional systems. J. Phys. C. 6, 1181 (1973).

25. Benenti, G., Waintal, X. \& Pichard, J.-L. New Quantum Phase between the Fermi Glass and the Wigner Crystal in Two Dimensions. Phys. Rev. Lett. 83, 1826-1829 (1999).

26. Spivak, B. \& Kivelson, S.A. Phases intermediate between a two-dimensional electron liquid and Wigner crystal. Phys. Rev. B 70, 155114 (2004).

27. Chen, Y.P. et al. Melting of a 2D quantum electron solid in high magnetic field. Nat. Phys. 2, 452-455 (2006).

28. Monarkha, Y.P. \& Syvokon, V.E. A two-dimensional Wigner crystal (Review Article). Low Temperature Physics 38, 1067-1095 (2012).

29. Cao, Y. et al. Correlated insulator behaviour at half-filling in magic-angle graphene superlattices. Nature 556, 80-84 (2018).

30. Cao, Y. et al. Unconventional superconductivity in magic-angle graphene superlattices. Nature 556, 43-50 (2018).

31. Tang, Y. et al. Simulation of Hubbard model physics in $\mathrm{WSe}_{2} / \mathrm{WS}_{2}$ moiré superlattices. Nature 579, 353-358 (2020).

32. Shimazaki, Y. et al. Strongly correlated electrons and hybrid excitons in a moiré heterostructure. Nature 580, 472-477 (2020).

33. Wang, L. et al. Correlated electronic phases in twisted bilayer transition metal dichalcogenides. Nat. Mater. 19, 861-866 (2020).

34. Cadiz, F. et al. Excitonic linewidth approaching the homogeneous limit in $\mathrm{MoS}_{2}$-based van der Waals heterostructures. Phys. Rev. X 7, 021026 (2017).

35. Ajayi, O.A. et al. Approaching the intrinsic photoluminescence linewidth in transition metal dichalcogenide monolayers. 2D Mater. 4, 031011 (2017).

36. Scuri, G. et al. Large excitonic reflectivity of monolayer $\mathrm{MoSe}_{2}$ encapsulated in hexagonal boron nitride. Phys. Rev. Lett. 120, 037402 (2018).

37. Back, P., Zeytinoglu, S., Ijaz, A., Kroner, M. \& Imamoğlu, A. Realization of an electrically tunable narrow-bandwidth atomically thin mirror using monolayer MoSe2. Phys. Rev. Lett. 120, 037401 (2018).

38. Cevallos, F.A. et al. Liquid salt transport growth of single crystals of the layered dichalcogenides $\mathrm{MoS}_{2}$ and $\mathrm{WS}_{2}$. Cryst. Growth Des. 19, $5762-5767$ (2019). 
39. Kadantsev, E.S. \& Hawrylak, P. Electronic structure of a single $\mathrm{MoS}_{2}$ monolayer. Solid State Commun. 152, 909-913 (2012).

40. Fallahazad, B. et al. Shubnikov--de Haas oscillations of high-mobility holes in monolayer and bilayer $\mathrm{WSe}_{2}$ : Landau level degeneracy, effective mass, and negative compressibility. Phys. Rev. Lett. 116, 086601 (2016).

41. Zarenia, M., Neilson, D., Partoens, B. \& Peeters, F.M. Wigner crystallization in transition metal dichalcogenides: A new approach to correlation energy. Phys. Rev. B 95, 115438 (2017).

42. Yoon, J., Li, C.C., Shahar, D., Tsui, D.C. \& Shayegan, M. Wigner crystallization and metal-insulator transition of two-dimensional holes in GaAs at $\mathrm{B}=0$. Phys. Rev. Lett. 82, 1744-1747 (1999).

43. Hossain, M.S. et al. Bloch ferromagnetism of composite fermions. Nat. Phys. (2020).

44. Mak, K.F. et al. Tightly bound trions in monolayer $\mathrm{MoS}_{2}$. Nature Mater. 12, 207-211 (2013).

45. Ross, J.S. et al. Electrical control of neutral and charged excitons in a monolayer semiconductor. Nat. Commun. 4, 1474 (2013).

46. Sidler, M. et al. Fermi polaron-polaritons in charge-tunable atomically thin semiconductors. Nature Phys. 13, 255-261 (2017).

47. Hubbard, J. Generalized Wigner lattices in one dimension and some applications to tetracyanoquinodimethane (TCNQ) salts. Phys. Rev. B 17, 494-505 (1978).

48. Kim, K. et al. van der Waals Heterostructures with high accuracy rotational alignment. Nano Lett. 16, 1989-1995 (2016).

49. Zhou, Y. et al. Controlling excitons in an atomically thin membrane with a mirror. Phys. Rev. Lett. 124, 027401 (2020).

50. Vil'k, Y.M. \& Monarkha, Y.P. Quasi-two-dimensional Wigner crystals with compound lattice. Fiz. Nizk. Temp. 10, 886-889 (1984).

51. Vil'nik, Y.M. \& Monarkha, Y.P. Structural phase transition in two-layer plane crystals with long-range interaction. Fiz. Nizk. Temp. 11, 971-982 (1985).

52. Bonsall, L. \& Maradudin, A.A. Some static and dynamical properties of a two-dimensional Wigner crystal. Phys. Rev. B 15, 1959-1973 (1977). 
53. Kormányos, A., Zólyomi, V., Drummond, N.D. \& Burkard, G. Spin-orbit coupling, quantum dots, and qubits in monolayer transition metal dichalcogenides. Phys. Rev. X 4, 011034 (2014).

54. Kormányos, A. et al. $\mathrm{k} \cdot \mathrm{p}$ theory for two-dimensional transition metal dichalcogenide semiconductors. 2D Mater. 2, 022001 (2015).

55. Larentis, S. et al. Large effective mass and interaction-enhanced Zeeman splitting of Kvalley electrons in MoSe2. Phys. Rev. B 97, 201407 (2018).

56. Platzman, P.M. \& Fukuyama, H. Phase diagram of the two-dimensional electron liquid. Phys. Rev. B 10, 3150-3158 (1974).

57. Goldoni, G. \& Peeters, F.M. Stability, dynamical properties, and melting of a classical bilayer Wigner crystal. Phys. Rev. B 53, 4591-4603 (1996).

58. Schweigert, I.V., Schweigert, V.A. \& Peeters, F.M. Enhanced stability of the square lattice of a classical bilayer Wigner crystal. Phys. Rev. B 60, 14665-14674 (1999).

59. Bedanov, V.M., Gadiyak, G.V. \& Lozovik, Y.E. On a modified Lindemann-like criterion for 2D melting. Phys. Rev. A 109, 289-291 (1985).

60. Imry, Y.\& Ma, S.-k. Random-field instability of the ordered state of continuous symmetry. Phys. Rev. Lett. 35, 1399-1401 (1975).

61. Ruzin, I.M., Marianer, S. \& Shklovskii, B.I. Pinning of a two-dimensional Wigner crystal by charged impurities. Phys. Rev. B 46, 3999-4008 (1992).

62. Fogler, M.M. \& Huse, D.A. Dynamical response of a pinned two-dimensional Wigner crystal. Phys. Rev. B 62, 7553-7570 (2000).

63. Chitra, R. \& Giamarchi, T. Zero field Wigner crystal. The European Physical Journal B Condensed Matter and Complex Systems 44, 455-467 (2005).

64. Spivak, B., Kravchenko, S.V., Kivelson, S.A. \& Gao, X.P.A. Colloquium: Transport in strongly correlated two dimensional electron fluids. Reviews of Modern Physics 82, 17431766 (2010).

65. Voelker, K. \& Chakravarty, S. Multiparticle ring exchange in the Wigner glass and its possible relevance to strongly interacting two-dimensional electron systems in the presence of disorder. Phys. Rev. B 64, 235125 (2001).

66. Waintal, X. On the quantum melting of the two-dimensional Wigner crystal. Phys. Rev. B 73, 075417 (2006). 
67. Falko, V.I. Optical branch of magnetophonons in a double-layer Wigner crystal. Phys. Rev. B 49, 7774-7777 (1994).

68. Hwang, E.H. \& Das Sarma, S. Plasmon dispersion in dilute two-dimensional electron systems: Quantum-classical and Wigner crystal--electron liquid crossover. Phys. Rev. B 64, 165409 (2001).

69. Knörzer, J. et al. Wigner crystals in two-dimensional transition-metal dichalcogenides: Spin physics and readout. Phys. Rev. B 101, 125101 (2020).

70. Tanatar, B. \& Ceperley, D.M. Ground state of the two-dimensional electron gas. Phys. Rev. $B$ 39, 5005-5016 (1989).

71. Zhu, X. \& Louie, S.G. Spin ordering of two-dimensional electron lattices. Phys. Rev. B 48, 13661-13665 (1993).

72. Bernu, B., Cândido, L. \& Ceperley, D.M. Exchange frequencies in the 2D Wigner crystal. Phys. Rev. Lett. 86, 870-873 (2001).

73. Xu, X., Yao, W., Xiao, D. \& Heinz, T.F. Spin and pseudospins in layered transition metal dichalcogenides. Nat. Phys. 10, 343-350 (2014).

74. Chen, Y.P. Pinned bilayer Wigner crystals with pseudospin magnetism. Phys. Rev. B 73, 115314 (2006).

75. Dayal, S., Clay, R.T., Li, H. \& Mazumdar, S. Paired electron crystal: Order from frustration in the quarter-filled band. Phys. Rev. B 83, 245106 (2011).

\section{Acknowledgments}

We acknowledge support from the DoD Vannevar Bush Faculty Fellowship (N00014-16-1-2825 for HP, N00014-18-1-2877 for PK), NSF CUA (PHY-1125846 for HP, ED, and MDL), Samsung Electronics (for HP and PK), NSF (PHY-1506284 for HP and MDL, DGE-1745303 for EB), AFOSR MURI (FA9550-17-1-0002), ARL (W911NF1520067 for HP and MDL), DOE (DESC0020115 for HP and MDL), and BME's TKP 2020 Nanotechnology grant (GZ). The device fabrication was carried out at the Harvard Center for Nanoscale Systems. This research used resources of the National Energy Research Scientific Computing Center (NERSC), a .U.S 
Department of Energy Office of Science User Facility operated under Contract No. DE-AC02$05 \mathrm{CH} 11231$.

\section{Author contributions}

H.P. and Y.Z. conceived the project. Y.Z., J.S., and E.B. fabricated the samples and designed/performed the experiments. I.E., Y.W., G.Z., and E.D. developed the theoretical model, and Y.Z., J.S., E.B., I.E., and Y.W. analyzed the data. G.S. assisted with optical measurements, R.G. assisted with sample fabrication, and H.H. grew the $\mathrm{MoSe}_{2}$ crystals. T.T. and K.W. provided hexagonal boron nitride samples. Y.Z., J.S., E.B., I.E., Y.W., G.S, E.D. and H.P. wrote the manuscript with extensive input from the other authors. H.P., E.D., P.K., and M.D.L. supervised the project.

\section{Competing financial interest}

The authors declare no competing financial interests. 


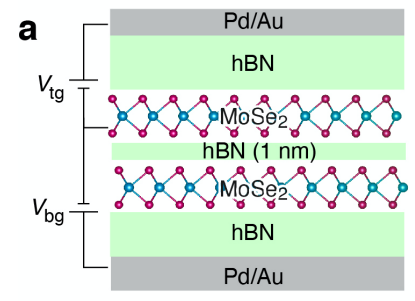

b

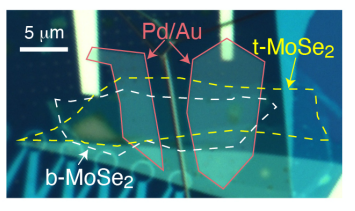

c

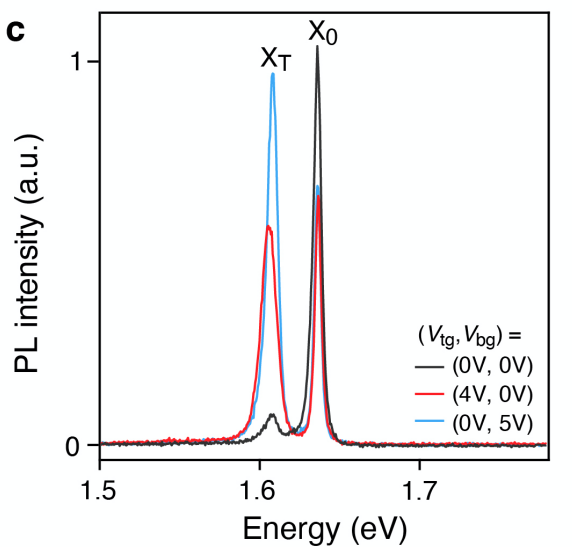

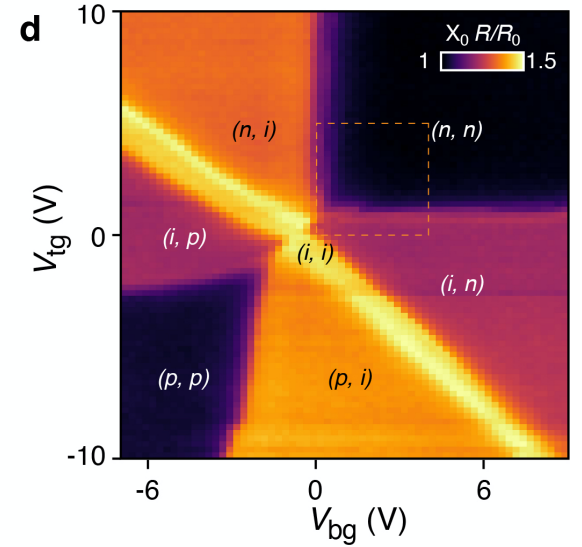

Figure 1. Device structure and full control of carrier density in the device. a, A schematic of the heterostructure cross section. The two $\mathrm{MoSe}_{2}$ monolayers are separated by a 1-nm thick layer of $\mathrm{hBN}$. The $\mathrm{MoSe}_{2} / \mathrm{hBN} / \mathrm{MoSe}_{2}$ structure is then encapsulated in $\mathrm{hBN}$ flakes of $15 \sim 20 \mathrm{~nm}$ thickness. b, An optical image of a $\mathrm{MoSe}_{2} / \mathrm{hBN} / \mathrm{MoSe}_{2}$ device. The top and bottom $\mathrm{MoSe}_{2}$ monolayer regions (t-MoSe $e_{2}$ and b-MoSe 2 , respectively) are indicated by the yellow and white dashed lines, respectively. The solid red lines show the outline of the optically transparent, thin top Pd/Au gates. c, Representative PL spectra of the $\mathrm{MoSe}_{2} / \mathrm{hBN} / \mathrm{MoSe}_{2}$ device measured at $4 \mathrm{~K}$ under different gate configurations. d, A 2D map of reflectance contrast from the neutral exciton $\mathrm{X}_{0}$ as a function of top $\left(V_{\mathrm{tg}}\right)$ and bottom $\left(V_{\mathrm{bg}}\right)$ gate voltages at $4 \mathrm{~K}$. We normalize the reflectance $R$ to its value at the highly-doped regime, $R_{0}$. From the strength of the reflectance, we can extract the charge states of the bilayer system. Here we denote the charge state $(t, b)$ in the order of top and bottom layer. $p, i$ and $n$ represent hole-doped, neutral and electron-doped, respectively. The dashed box represents the voltage range we focus on in later studies. 

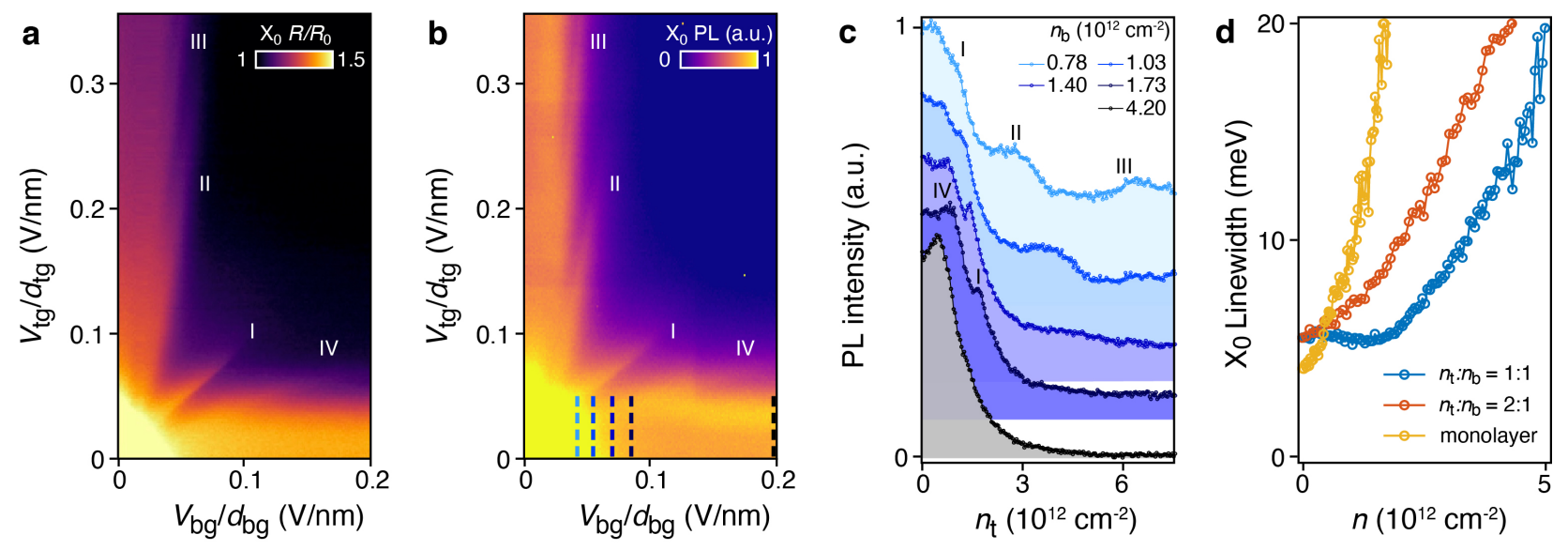

Figure 2. Voltage-dependent reflectance and PL spectra of a hBN/ $\mathrm{MoSe}_{2} / \mathrm{hBN} / \mathrm{MoSe}_{2} / \mathrm{hBN}$ device in the electron doped regime at $4 \mathrm{~K}$. a-b, 2D maps of neutral exciton $\mathrm{X}_{0}$ reflectance contrast $R / R_{0}$ (a) and integrated $\mathrm{X}_{0} \mathrm{PL}(\mathbf{b})$, as a function of $V_{\mathrm{tg}} / d_{\mathrm{tg}}$ and $V_{\mathrm{bg}} / d_{\mathrm{bg}}$. Here we divide the top and bottom gate voltages by the respective hBN thicknesses so that the slopes of the linear features correspond to the ratios of the electrostatically induced carrier densities in each layer, $n_{\mathrm{t}}: n_{\mathrm{b}}$. Features denoted as I, II, III, and IV can be clearly observed in these maps. c, The integrated $\mathrm{X}_{0}$ PL intensity as a function of $n_{\mathrm{t}}$, while $n_{\mathrm{b}}$ is kept as a constant. The lower bound of the shaded region represents zero PL intensity for each curve. The corresponding bottom gate voltages for these linecuts are indicated by the dashed lines in $\mathbf{b}$. $\mathbf{d}$, The linewidth of the neutral exciton as a function of total electron density $n$, measured along $n_{\mathrm{t}}: n_{\mathrm{b}}=1: 1$ and 2:1, compared with a monolayer $\mathrm{MoSe}_{2}$. 

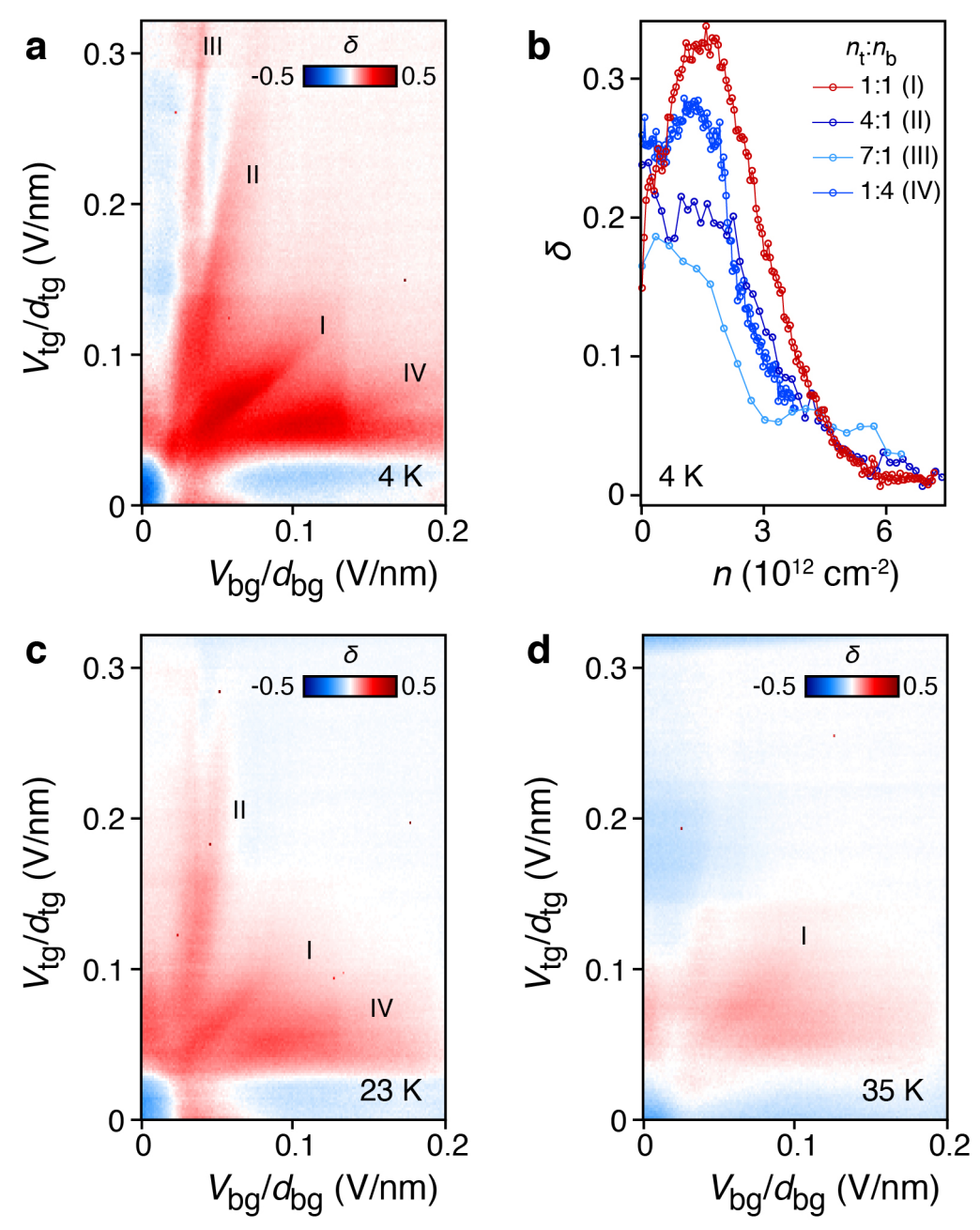

Figure 3. Density- and temperature-dependence of the interaction-induced insulating states. a, A 2D map of $\delta\left(n_{\mathrm{t}}, n_{\mathrm{b}}\right)$, which characterizies the formation of the interaction-induced insulating states, as a function of $V_{\mathrm{tg}} / d_{\mathrm{tg}}$ and $V_{\mathrm{bg}} / d_{\mathrm{bg}}$ at $4 \mathrm{~K} . \mathbf{b}, \delta$ as a function of the total electron density in both layers, $n=n_{\mathrm{t}}+n_{\mathrm{b}}$, for various features, i.e. $n_{\mathrm{t}}: n_{\mathrm{b}}=1: 1,4: 1,7: 1$, and 1:4 at $4 \mathrm{~K}$. c-d, 2D maps of $\delta\left(n_{\mathrm{t}}, n_{\mathrm{b}}\right)$ as a function of $V_{\mathrm{tg}} / d_{\mathrm{tg}}$ and $V_{\mathrm{bg}} / d_{\mathrm{bg}}$ at $\mathbf{c}, 23 \mathrm{~K}$, and $\mathbf{d}, 35 \mathrm{~K}$. 

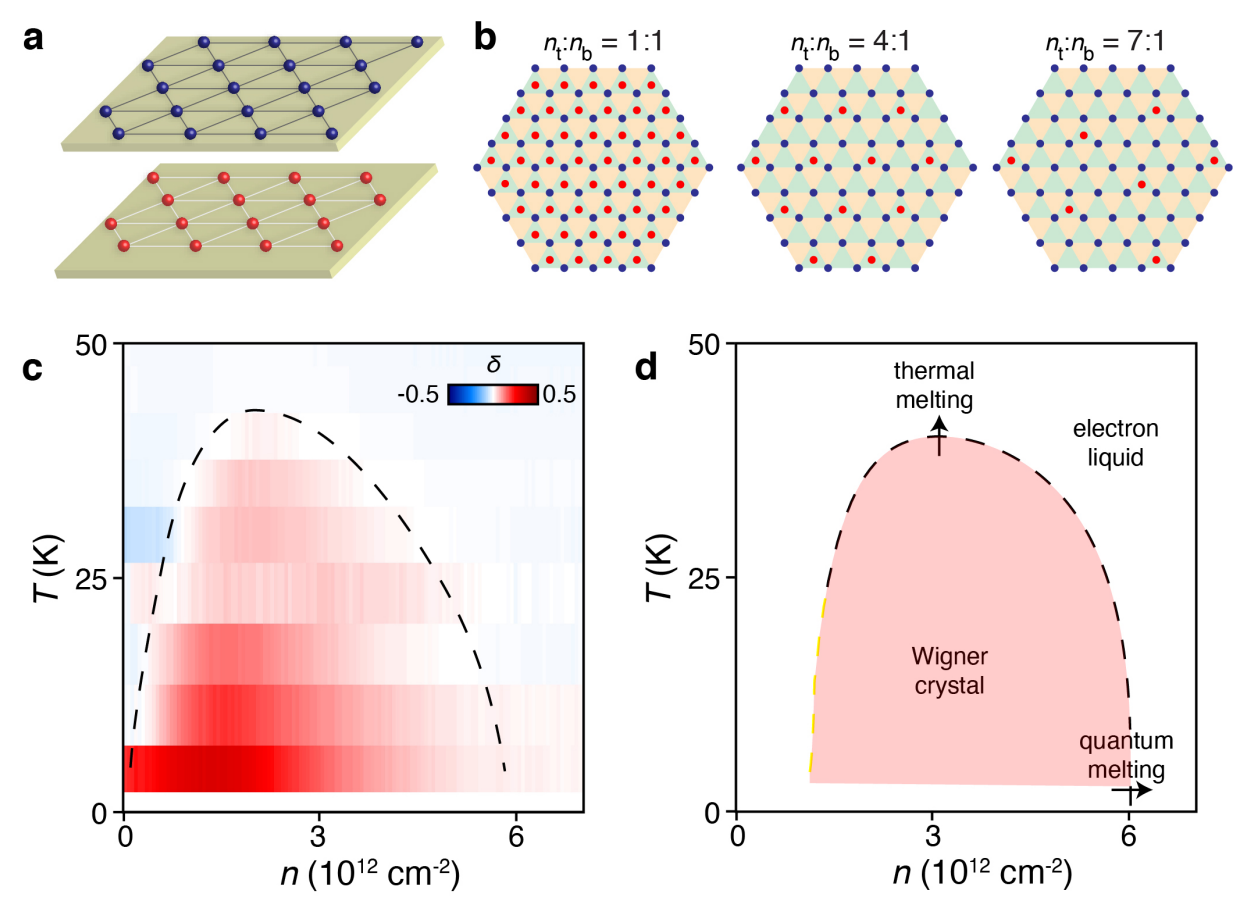

Figure 4. Bilayer Wigner crystals and their quantum and thermal phase transitions. a, A schematic of Wigner crystal in bilayer systems. b, Schematics of commensurate stacking in bilayer Wigner crystals with triangular lattices for filling ratios $n_{\mathrm{t}}: n_{\mathrm{b}}$ of $1: 1,4: 1$, and 7:1. c, A 2D map of $\delta\left(n_{\mathrm{t}}, n_{\mathrm{b}}\right)$ as a function of total carrier density $n$ and temperature $T$ for $n_{\mathrm{t}}: n_{\mathrm{b}}=1: 1$. The $\delta>0$ region represents where the bilayer Wigner crystal forms (the $\delta=0$ boundary shown as a dashed line). The center position of each pixel in the $y$ axis represents the temperature. d, A schematic phase diagram of bilayer Wigner crystals, showing both quantum and thermal phase transitions. The dashed line represents a calculated melting curve assuming Lindenman parameter $\gamma \sim 0.56$. Below a density of $\sim 1.4 \times 10^{12} \mathrm{~cm}^{-2}$, theory predicts the staggered triangular lattice structure becomes unstable (green dashed line). 


\section{Methods:}

\section{Device fabrication}

Monolayer $\mathrm{MoSe}_{2}$ and hBN flakes were exfoliated from bulk crystals onto silicon substrates with a 285-nm silicon oxide layer. Monolayers of $\mathrm{MoSe}_{2}$ were identified under an optical microscope and verified via photoluminescence measurements. The thickness of hBN flakes was measured by an atomic force microscope. Then we fabricated the $\mathrm{hBN} / \mathrm{MoSe}_{2} / \mathrm{hBN} / \mathrm{MoSe}_{2} / \mathrm{hBN}$ heterostructure using a tear-and-stack technique with a dry transfer method; a monolayer of $\mathrm{MoSe}_{2}$ was torn into two pieces which were then stacked without introducing any rotation between them. This heterostructure was then transferred onto the bottom gate (1 nm $\mathrm{nr}$ and $9 \mathrm{~nm} \mathrm{Pd} / \mathrm{Au}$ alloy), prepatterned by electron-beam lithography and thermal evaporation. Then top gates $(1 \mathrm{~nm} \mathrm{Cr}$ and 9 $\mathrm{nm} \mathrm{Pd} / \mathrm{Au}$ alloy) were defined with electron-beam lithography and deposited via thermal evaporation. Finally, we made electrical contacts to the $\mathrm{MoSe}_{2}$ and the gates using $5 \mathrm{~nm} \mathrm{Cr}$ and 90 $\mathrm{nm}$ Au deposited via thermal evaporation to connect them to the wire-bonding pads.

\section{Optical spectroscopy}

Optical measurements were carried out in a home-built confocal microscope with a $4 \mathrm{~K}$ cryostat from Montana Instruments. The apochromatic objective used has a numerical aperture of 0.75 . We excited the sample using a 660-nm wavelength diode laser with various power for the PL measurements. We obtained the reflectance spectra by illuminating the sample with a broadband light source, using a halogen lamp (Thorlabs SLS201L) and a supercontinuum laser from NKT Photonics. The spectra were measured by a spectrometer using either a 300 line $/ \mathrm{mm}$ or a 1200 line/mm grating and a Princeton Instruments camera (PIXIS 2048). The gate voltages were supplied by two Keithley 2400 sourcemeters. 
Data availability. The data that support the plots within this paper and other findings of this study are available from the corresponding author upon reasonable request. 